\title{
The Research on the Quick Discharge Circuit of Shunt Capacitor
}

\author{
Shuheng Dan, Lei Ning \\ Department of Electrical Engineering, Shanghai University of Electric Power, Shanghai, China \\ Email: danshuheng@shiep.edu.cn
}

Received November 2013

\begin{abstract}
Shunt capacitor is the main method to intensively compensate reactive power in the power system. The external discharge circuit, commonly discharge coil which is an inductance coil with low resistance, must be allocated to the large capacity of capacitor. This paper calculates and compares two kinds of circuit, capacitor placed with a discharge coil or a resistance, to find the best way to discharge the residual charge of capacitor. Through the study, in the under damped case, these two kinds of circuit present different discharge properties. In over damped mode, their discharge properties are similar.
\end{abstract}

\section{Keywords}

Discharge Coil; Discharge Property; Capacitor

\section{Introduction}

In the $66 \mathrm{kV}$ and below power system, discharge coil shunted with high-voltage capacitors quickly release the residual charge of the capacitor as it removed from the power system to ensure the security of the capacitor itself and other devices when they are put into use again [1]. The whole process of discharge can be equaled with RLC circuit in which charged capacitance $\mathrm{C}$ interact with inductance $\mathrm{L}$ and resistor $\mathrm{R}$ [2]. The combination of the three parameters may result in oscillation which should be avoided in actual operation [3]. In the process of discharge, the RC circuit without inductance L can not only prevent the phenomenon of oscillation, but also reach the purpose of releasing energy. This paper compared the discharge property of the two circuits by observing time $t$, during which the voltage of capacitor decays to a curtain value, and the variation of the current.

\section{The Mathematical Model}

The power industry standard DL/T653-2009-HV shunt capacitor discharge coil ordering technology [4] makes a clear regulation of the discharge property of discharge coil. The voltage of the capacitor removed from the power system drops from $\sqrt{2} U_{n}$ to $50 \mathrm{~V}$ in $5 \mathrm{~s}$.

After the RLC equivalent circuit switch closed, the whole process is equal to zero input response of the second-order circuit. In order to make sure the changing circumstance of the voltage $U_{c}$ which is between the two ends of the capacitor, the second order constant coefficient differential equation with its initial conditions of 
the equivalent circuit depicted in Figure 1 should be given based on the knowledge of circuit, like in Equation (1).

$$
\begin{aligned}
& L C \frac{d_{U_{c}}^{2}}{d t^{2}}+R C \frac{d U_{c}}{d t}+U_{c}=0 \\
& U_{c}\left(0_{+}\right)=U_{0} \\
& \left.\frac{d U_{c}}{d t}\right|_{t=0_{+}}=0
\end{aligned}
$$

Due to the relation between the circuit resistance $\mathrm{R}$ and the critical resistance $R_{\mathrm{cr}}=2 \sqrt{L / C}$, the situation of $U_{c}$ can be divided into four kinds of circumstance, over damped non-oscillation, Critically damped non-oscillation, under damped oscillation and undamped oscillation.

Because the remaining energy cannot be consumed the undamped oscillation circuit is not satisfied with the demand of the standard. The other three kinds of solution are described in the following Equations (2)-(4).

$$
\begin{gathered}
U_{c}(t)=U_{0}(1+\delta t) e^{-\delta t} \\
U_{c}(t)=\frac{U_{0}}{s_{2}-s_{1}}\left(s_{2} e^{s_{1} t}-s_{1} e^{s_{2} t}\right) \\
U_{c}(t)=\frac{\omega_{0} U_{0}}{\omega} e^{-\delta t} \sin (\omega t+\beta)
\end{gathered}
$$

where,

$$
\begin{aligned}
& \delta=R /(2 L) \\
& S_{1,2}=-\delta \pm j \omega \\
& \omega_{0}=\sqrt{1 /(L C)} \\
& \omega=\sqrt{1 /(L C)-(R /(2 L))^{2}}
\end{aligned}
$$

It is clear that if the Equation (4) wants to meet the need of the standard, the coefficient, which is $\left(\omega_{0} U_{0}\right) \cdot e^{-\delta t} / \omega$, should decays to $50 \mathrm{~V}$ in time. Equation (5) is a simplified formula.

$$
t_{R L C}=\frac{2 L}{R} \ln \left(\frac{U_{0}}{50} \cdot \frac{1}{\sqrt{1-\frac{R^{2} C}{4 L}}}\right)
$$

Figure 2 shows a RC circuit. After the switch closed, the capacitor C interacts with the resistance R. The whole process is equal to zero input response of the first-order circuit.

The first order constant coefficient differential equation and the initial conditions of the RC equivalent circuit are given in Equation (6).

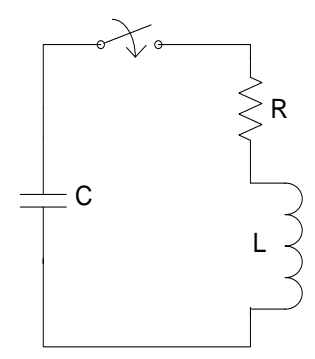

Figure 1. RLC equivalent circuit. 


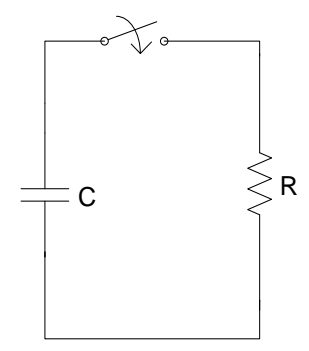

Figure 2. RC equivalent circuit.

$$
\begin{aligned}
& R C \frac{d U_{c}}{d t}+U_{c}=0 \\
& U_{c}\left(0_{+}\right)=U_{0}
\end{aligned}
$$

And the solution is Equation (7)

$$
U_{c}(t)=U_{0} e^{-\frac{t}{R C}}
$$

Letting the right side of Equation (7) equals to 50, the time $t$ in which the voltage of the capacitor drops to 50 $\mathrm{V}$ can be got in Equation (8).

$$
t_{R C}=R C \ln \frac{U_{0}}{50}
$$

\section{The Analysis and Calculation of the Mathematical Model}

The type of capacitor is BW10.5-12-1 and its capacitance is $0.347 \mu \mathrm{F}$. The reductive reactance in circuit is $12 \%$ of the capacitive reactance. Because of the uncertainty of the solution of the second order constant coefficient differential equation the comparison of the discharge property of the two kinds of circuit must be discussed separately.

The critical resistance $R_{\text {cr }}$ equaled to $2 \sqrt{L / C}$ in RLC circuit is determined by capacitance C and inductance L. Assuming that the circuit resistance R is a variable, the situation in RLC circuit can be analyzed in the situations of over damping, under damping and critical damping based on the relation between the circuit resistance and the critical resistance. In RC circuit, the size of resistance R can also be classified according to this standard.

\subsection{Critically Damped Case}

In this case, the circuit resistance of the RLC and RC circuit is both equal to critical resistance. Equations (2) and (7) are both monotonous attenuation functions. In order to get their discharge property, the curves of the voltage of the capacitor can be drawn separately in Figure 3.

Figure 3 shows that at the beginning the discharge rate of RC circuit is higher than RLC circuit, nevertheless the situation then changes as the voltage decays to a certain value. Compared with the RC circuit, the voltage of the RLC circuit uses less time to reach the established value.

\subsection{Under Damped Case}

The resistance of the two circuits here is smaller than critical resistance. Figure 4, in which the resistance $\mathrm{R}$ is a variable, shows the curves of voltage-decaying in under damped state. Time $t_{R C}$ which stands for the duration of voltage-decaying in RC circuit increases as the resistance increases. But for the RLC circuit, time $t_{R L C}$ decreases at first and then increase as the resistance increases. Considering this mathematical model, time $t_{R L C}$ is significantly greater than the time $t_{R C}$ as the resistance changes in the range of a few hundred ohms. With the value of resistance keeping increasing and reaching $0.731 R_{\text {cr }}(4647 \Omega)$ which is the maximum of the range, time $t_{R L C}$ is greater than time $t_{R C}$ all the time, but the gap is constantly narrowed. When the circuit resistance 


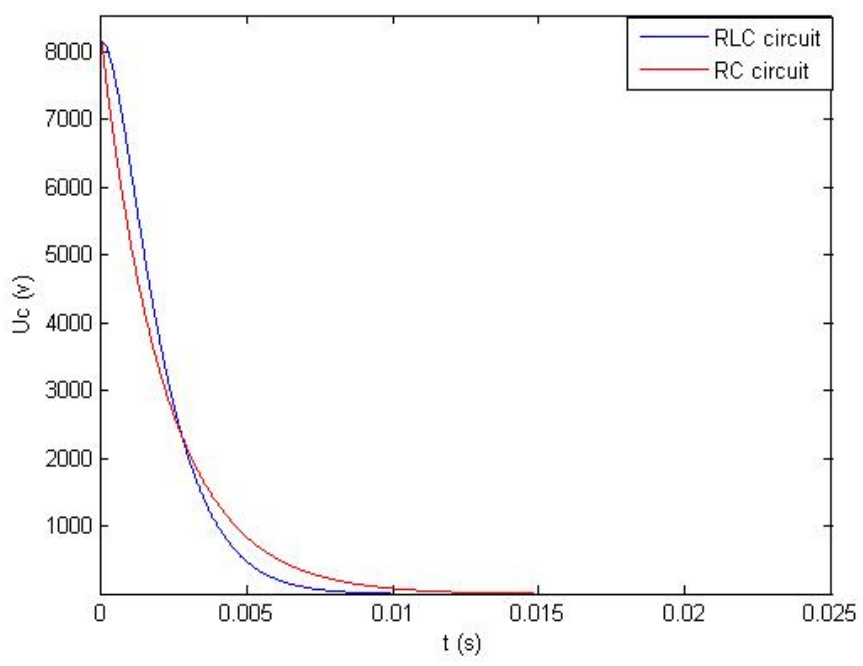

Figure 3. Voltage-decaying in critically damped case.

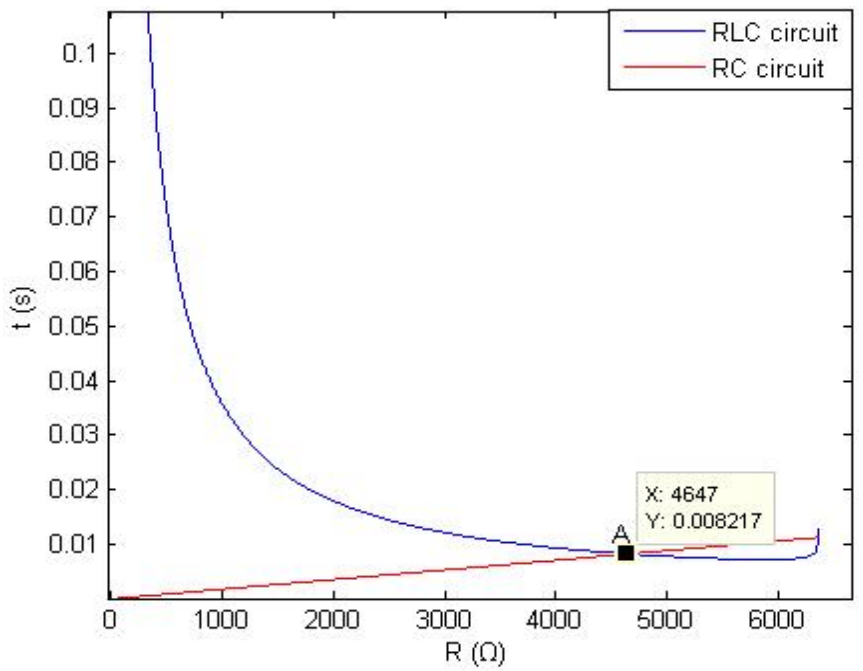

Figure 4. Time for voltage-decaying to 50V in under damped case.

changes in the range $\left(0.731 R_{\mathrm{cr}}, R_{\mathrm{cr}}\right)$, time $t_{R L C}$ is smaller than time $t_{R C}$. In addition, these two curves have a tendency to intersect at the critical resistance.

\subsection{Over Damped Case}

The resistance of the two kinds of circuit is larger than critical resistance. Equation (3) established the voltagedecaying of the RLC circuit in over damped non-oscillation. Given by the equation, $U_{c}$ is a superposition of two items of exponential decay and the capacitor is discharging all the time.

Figure 5 is the curves of Equations (3) and (8) and the relation between the voltage-decaying time and the variable R. From this figure, the time $t$ increases as the circuit resistance increases and the two lines are less distinct.

The solutions of Equations (1) and (6) can be calculated by MATLAB. Adjusting the size of the resistance in the circuits the series of voltage-decaying time can be obtained in Table 1. And the first column is the ratio of circuit resistance and critical resistance.

In Table 1, in RLC circuit time $t$ decreases at first and then increases as the circuit resistance increases consistently. However, time $\mathrm{t}$ in RC circuit is increases all through. Compared with the two columns of Table 1, time $\mathrm{t}$ is similar when the circuit resistance is above $R_{c r}$. Take $5 R_{c r}$ as an example depicted in Figure 6. 


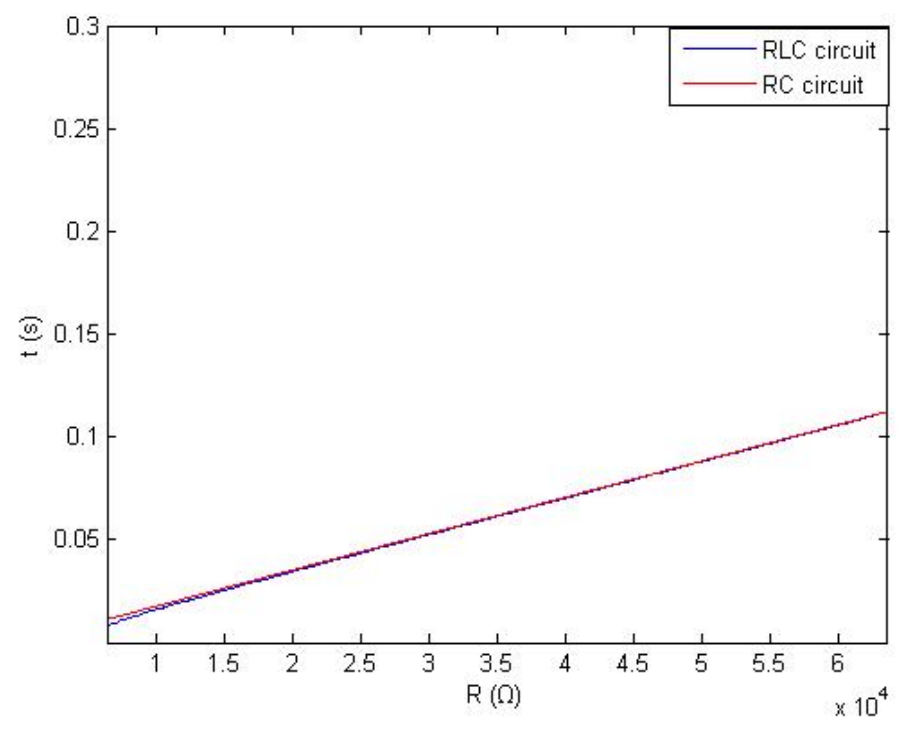

Figure 5. Time for voltage-decaying to $50 \mathrm{~V}$ in over damped case.

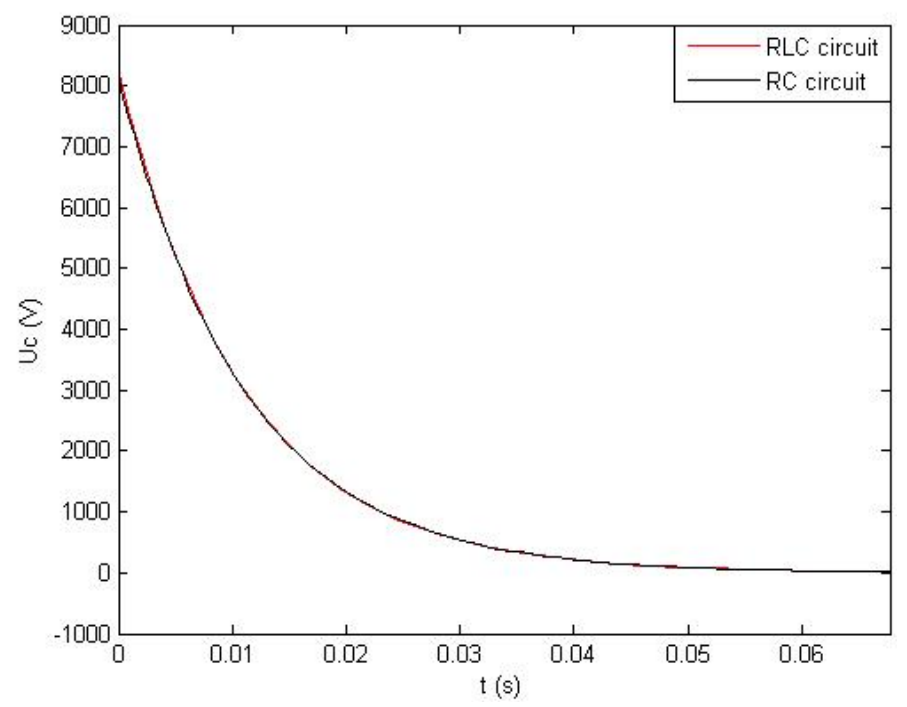

Figure 6. Voltage-decaying in $5 R_{c r}$.

Table 1. Time for voltage-decaying to $50 \mathrm{~V}$ with variable circuit resistance.

\begin{tabular}{ccc}
\hline Ratio $R / R_{c r}$ & \multicolumn{2}{c}{ Time for voltage decaying to $50 \mathrm{~V} \mathrm{t} / \mathrm{ms}$} \\
\cline { 2 - 3 } 5 & RLC circuit & RC circuit \\
\hline 2 & 54.84 & 57.08 \\
1 & 21.5 & 22.83 \\
0.9 & 8.03 & 11.42 \\
0.7 & 5.9 & 10.27 \\
0.5 & 7.7 & 7.99 \\
0.1 & 10.04 & 5.71 \\
\hline
\end{tabular}




\subsection{The Change of Circuit Current}

Figure 7 is a variation of circuit current when the circuit resistance equals to $0.731 R_{c r}$. The amplitude of the current in RC circuit is higher, which is about 1.6 times of RLC circuit's value, meanwhile the duration of voltage-decaying to $50 \mathrm{~V}$ of both circuits is same.

It is a common sense that excess current may influences the normal operation, or even destroys devices. Figure 8, in which $\mathrm{x}$-coordinate is the ratio of circuit resistance and critical resistance, shows the relation between the current peak and circuit resistance. The amplitude of both circuits is decreases as the circuit resistance increases. And the peak value of RLC is smaller than RC's.

\section{Conclusions}

This paper analyzes the property of a capacitor assembled with a discharge coil and a resistance. And some general conclusions can be obtained based on these results.

1) In under damped case, in RLC circuit the voltage-decaying time decreases at first and then increases as the circuit resistance increases; in RC circuit the time increases consistently. In over damped case, the trend of the voltage-decaying in two circuits is similar.

2) The amplitude of the current in both circuits goes down as the resistance increases, and the peak value of current in RLC circuit is smaller than RC's all the time.

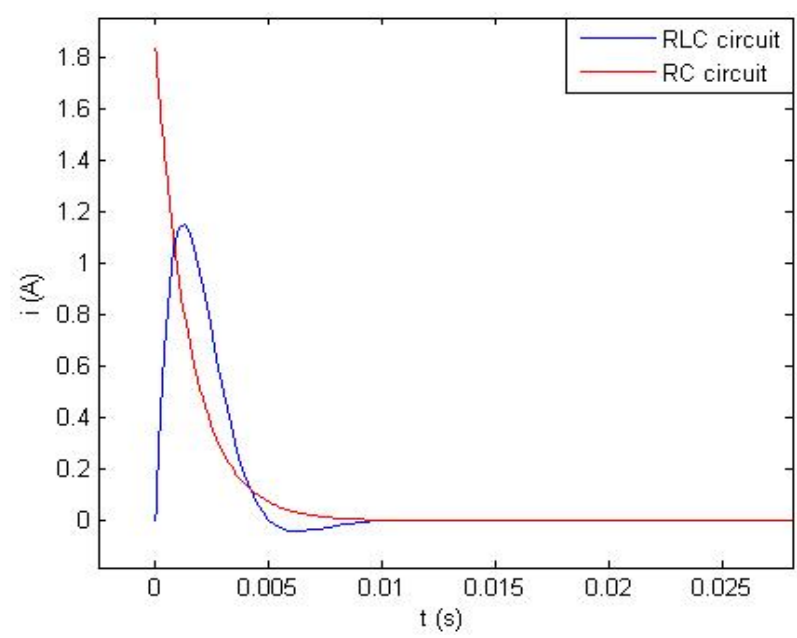

Figure 7. Current in two circuits with $R$ equaled to $0.731 R_{c r}$.

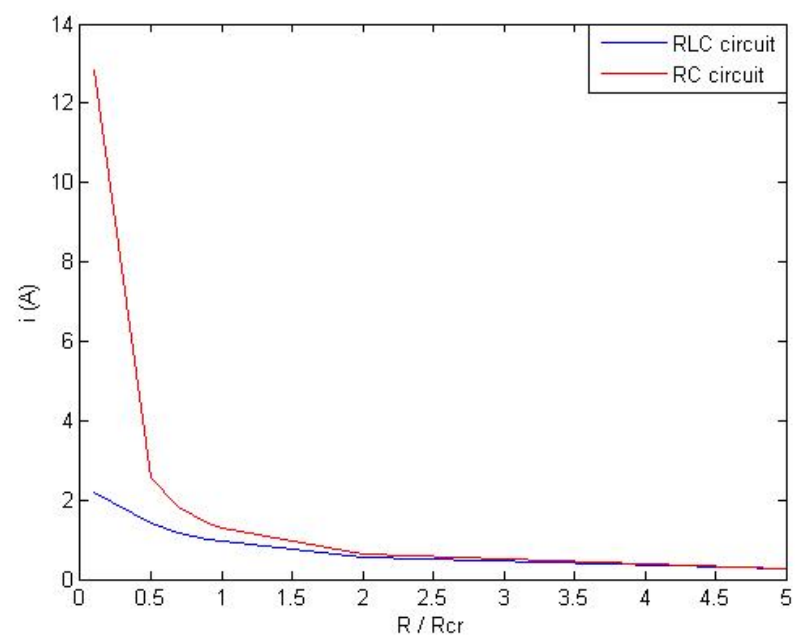

Figure 8. The current peak with the variable ratio $R / R_{c r}$. 
3) Considering of actual operation, discharge circuit should limit the size of discharge time and the current peak well. On this occasion defined by minimum discharge time and smaller current peak, the property of discharge coil is better than resistance.

\section{Acknowledgements}

I owe appreciation to the fund of national nature science and the Shanghai Municipal Education Commission. This paper is financially supported by the Fund of National Nature Science, project number is 50577040. And supported by Leading Academic Discipline Project of Shanghai Municipal Education Commission, project number is J51303.

\section{References}

[1] Zhang, H.L., Huang, X.M. and Wu, Y.M. (2010) Selection of Discharge Coil for High Voltage Shunt Capacitor Bank. Power Capacitor and Reactive Power Compensation, 31, 26-29.

[2] Zhou, G.L. (2000) Test and Research on Characteristics of Discharge Coils for High Voltage Shunt Capacitors. Zhejiang Electric Power, 5, 7-10.

[3] Wu, B. and Wang, X.J. (2006) Research on the Parameters of Discharge Coil of Shunt Capacitor Bank. Tianjin Electric Power Technology, 4, 35-37.

[4] The National Energy Administration of China (2009) DL/T653-2009 HV Shunt Capacitor Discharge Coil Ordering Technology. China Electric Power Express, Beijing. 